\title{
Asymptotically consistent size-dependent plate models based on the couple-stress theory with micro-inertia
}

\author{
Andrea Nobilia \\ ${ }^{a}$ Department of Engineering "Enzo Ferrari", University of Modena and Reggio Emilia, via \\ Vivarelli 10, 41125 Modena, Italy
}

\begin{abstract}
Several beam and plate models have been recently developed in the literature to accommodate for size-dependence. These are usually obtained starting from a generalized continuum theory (such as the couple-stress, strain-gradient or nonlocal theory or their modifications) and then deducing the governing equations through Hamilton's principle and ingenuous kinematical assumptions. This approach, originated by Kirchhoff, usually fails to reproduce the dispersion features of the equivalent 3D theory. Besides, it produces a variety of models, in dependence of the different assumptions, such as Kirchhoff's or Mindlin's. In contrast, in this paper we adopt asymptotic reduction: moving from the couple-stress linear theory of elasticity with micro-inertia, we deduce new models for elongation and flexural deformation of microstructured plates. The resulting models are consistent, in the sense that they reproduce the dispersion features of the corresponding $3 \mathrm{D}$ body. Also, models are unique, for they may only differ by the order of the approximation. We find that microstructure especially affects inertia terms, which can be hardly captured by a-priori kinematical assumptions. For static flexural deformations, our results match those already obtained assuming plane cross-sections within the modified couple-stress theory. In fact, we show that couple-stress, reduced couple-stress and strain gradient theories all lead to equivalent results. Higher order models are also given, that describe the near first-cut-off behaviour and account for thickness deformations in the
\end{abstract}

* Corresponding author

Preprint submitted to Elsevier

April 16, 2021 
spirit of Timoshenko.

Keywords: Asymptotic model, Microstructured plate, Couple stress, Rayleigh-Lamb waves

\section{1. Introduction}

$2 \quad$ Modern technology heavily relies on micro- and nano-components for sens3 ing, actuating, filtering and testing. It is an established experimental fact that 4 mechanical response of these structures depends on their scale (Chong et al. 5 2001, Radi et al. 2020). However, the classical theory of elasticity lacks an inter-

6 nal length-scale and it is therefore incapable of incorporating this dependence.

7 Precisely to remedy this deficiency, which leads to several limitations Nobili

8 et al. 2020), many generalized continuous theories (GCT) have been proposed, 9 such as surface elasticity (Gurtin and Murdoch, 1975), non-local elasticity (Erin10 gen, 1984) and strain gradient theories (Yang et al., 2002). Couple stress theory

11 is perhaps the simplest micropolar theory (Toupin, 1962, Koiter, 1969), where an extra rotational displacement is considered (the micro-motion), that is related to the skew symmetric part of the displacement (the macro-motion) gradient. In this respect, it is also a strain gradient theory. Recently, adaptations of the original theory have been proposed, in an attempt to reduce the number of material parameters, such as the modified or reduced couple stress theory Yang et al. 2002), which postulates a new equilibrium equation for the moment of momenta, the symmetric theory (Hadjesfandiari and Dargush, 2011), which restricts the curvature tensor to being symmetric, and the strain gradient effect, originating from plasticity and relating to geometrically necessary dislocations (Fleck and Hutchinson, 1993). Also, the majority of studies concerning couple stress (CS) theory neglects the role of micro-rotational inertia, owing to the increased mathematical complications.

In this context, a large number of contributions has recently appeared in the literature introducing new dimensional-reduced models, such as beams, plates, shells, incorporating microstructural features. The generality of these is ob- 
tained moving from an enhanced continuum theory and then deducing the new equilibrium equations through an application of Hamilton's principle, in conjunction with ingenuous kinematical constraints. This is indeed the method originally adopted by Kirchhoff (1859) in developing the classical plate theory that bears his name, and later extended to shells by Love, see (Love, 1888) and the excellent historical introduction therein. Along these lines, Ma et al. (2008) developed a Timoshenko-like beam model moving from the differential nonfocal model of Eringen, which was later found to lead to paradoxes Mikhasev and Nobili (2020). In Park and Gao (2006), a static Bernoulli-Euler theory of size-dependent beams is introduced from the reduced couple stress (RCS) theory by the minimum energy principle. Tsiatas (2009) developed a static Kirchhoff plate model based on the RCS theory, which was later extended to dynamics by Yin et al. (2010) simply by addition of translational inertia. On a similar basis, Jomehzadeh et al. (2011) introduced size-dependence in micro-plates, accounting for plate extension and adding rotational inertia. Mindlin-like models, where cross-section rotation is an extra degree of freedom, have been presented in Ma et al. (2011); Zhou and Gao (2014), again moving from the modified couple stress theory. Similar contributions, but departing from the strain gradient (SG) theory, are presented in Lazopoulos (2004) and, successively, in Lazopou$\operatorname{los}(2009)$ with the addition of surface elasticity, both being restricted to statics and arriving at a higher-order plate theory.

In this paper, we develop a novel size-dependent plate model by asymptotic reduction of the elastodynamics of a thin strip of couple stress elastic isotropic material with micro-inertia, encompassing for extension and flexure. As described by Kaplunov et al. (1998) in their excellent monograph, asymptotic reduction is a powerful technique by which dimensional-reduced models are built which preserve the fundamental dispersion feature of the original 3D body. Precisely in this sense, models are defined consistent. Models are also unique, for a given range of approximation (say, long-wave low-frequency). Higher order (or, better, long-wave high-frequency) models are also constructed, which reproduce dispersion near the first cut-on frequency (the first overtone) and add 


\section{2. Fundamentals of couple stress theory}

In a linear elastic couple stress solid, the traditional displacement field $\boldsymbol{u}$ is supplemented by the rotation vector $\boldsymbol{\varphi}$, which represents the motion at the micro-level. In contrast to micropolar theories, the latter is related to the former through

$$
\varphi=\frac{1}{2} \operatorname{curl} \boldsymbol{u}
$$

We introduce the classical measure of deformation (strain tensor)

$$
\varepsilon=\text { Sym grad } \boldsymbol{u}
$$

alongside the torsion-flexure or curvature tensor, which is typical of higher gradient theories (the wryness tensor)

$$
\chi=\operatorname{grad} \varphi
$$

For an isotropic hyperelastic material, we introduce the stored elastic potential $U=U(\varepsilon, \boldsymbol{\chi})$ and obtain the constitutive equations

$$
\begin{array}{lll}
\boldsymbol{\sigma}=\frac{\partial U}{\partial \varepsilon} & \Rightarrow & \boldsymbol{\sigma}=2 G \varepsilon+\Lambda(\operatorname{tr} \varepsilon) \mathbf{1} \\
\boldsymbol{\mu}=\frac{\partial U}{\partial \chi} & \Rightarrow & \boldsymbol{\mu}=2 G \ell^{2}\left(\chi^{T}+\eta \chi\right)
\end{array}
$$

64 where 1 is the identity tensor and $\ell>0,-1<\eta<1$ is a pair of material ${ }_{65}$ constants connected to the microstructure, while $G$ and $\Lambda$ are the traditional

${ }_{66}$ Lamé parameter. Eqs.(1) specify the symmetric part of the stress tensor, $\boldsymbol{\sigma}=$ ${ }_{67}$ Sym $\boldsymbol{t}$, and the couple stress tensor $\boldsymbol{\mu}$, respectively. The latter is a deviatoric 
69 gradient effect.

The equations of motion express equilibrium of linear and angular momentum

$$
\begin{aligned}
\operatorname{div} \boldsymbol{t} & =\rho \ddot{\boldsymbol{u}}, \\
2 \operatorname{axial} \boldsymbol{t}+\operatorname{div} \boldsymbol{\mu} & =J \ddot{\boldsymbol{\varphi}},
\end{aligned}
$$

71 where $\rho$ is the mass density and $J \geq 0$ is the micro-inertia, with physical diand $(\text { axial } \boldsymbol{t})_{i}=e_{i j k} t_{k j}$ denotes the axial vector attached to any skew symmetric tensor (here $e_{i j k}$ is the permutation tensor).

Hereinafter, we restrict our analysis to plane-strain conditions

$$
u_{1}\left(x_{1}, x_{2}, t\right), \quad u_{2}\left(x_{1}, x_{2}, t\right), \quad u_{3}\left(x_{1}, x_{2}, t\right)=0,
$$

whence, the non-zero components of $\varepsilon, \varphi$ and $\chi$ read

$$
\begin{aligned}
\varepsilon_{11} & =u_{1,1}, & \varepsilon_{12}=\frac{1}{2}\left(u_{1,2}+u_{2,1}\right)=\varepsilon_{21}, & \varepsilon_{22}=u_{2,2}, \\
\varphi_{3} & =\frac{1}{2}\left(u_{2,1}-u_{1,2}\right), & & \\
\chi_{31} & =\frac{1}{2}\left(u_{2,11}-u_{1,21}\right), & \chi_{32} & =\frac{1}{2}\left(u_{2,12}-u_{1,22}\right) .
\end{aligned}
$$

Together, Eqs. (4) with the constitutive equations (1) give

$$
\begin{array}{rlrl}
\sigma_{11} & =2 G u_{1,1}+\Lambda\left(u_{1,1}+u_{2,2}\right), & & \sigma_{22}=2 G u_{2,2}+\Lambda\left(u_{1,1}+u_{2,2}\right), \\
\sigma_{33} & =\Lambda\left(u_{1,1}+u_{2,2}\right), & & \\
\sigma_{12} & =\sigma_{21}=G\left(u_{1,2}+u_{2,1}\right), & & \\
\mu_{13} & =G \ell^{2}\left(u_{2,11}-u_{1,21}\right), & \mu_{23}=G \ell^{2}\left(u_{2,12}-u_{1,22}\right), \\
\mu_{31}=G \ell^{2} \eta\left(u_{2,11}-u_{1,21}\right), & \mu_{32}=G \ell^{2} \eta\left(u_{2,12}-u_{1,22}\right),
\end{array}
$$

In plane strain, Eqs. $(2)$ become

$$
\begin{aligned}
\sigma_{11,1}+\sigma_{21,2}+\tau_{21,2} & =\rho \ddot{u}_{1}, \\
\sigma_{12,1}+\tau_{12,1}+\sigma_{22,2} & =\rho \ddot{u}_{2}, \\
2 \tau_{12}+\mu_{13,1}+\mu_{23,2} & =J \ddot{\varphi}_{3},
\end{aligned}
$$


76

$$
\tau_{12}=-\tau_{21}=-\frac{1}{2} G \ell^{2}\left(\hat{\triangle} u_{2,1}-\hat{\triangle} u_{1,2}\right)+\frac{J}{4}\left(\ddot{u}_{2,1}-\ddot{u}_{1,2}\right) .
$$

Here, the notation $\hat{\triangle}$ indicates the 2-D Laplace operator in the $x_{1}, x_{2}$ coordinates. Substituting (5) and (7) into (6a) and (6b) leads to the following expression for the governing equations in terms of displacement

$$
\begin{aligned}
(\Lambda+G)\left(u_{1,11}+\right. & \left.u_{2,12}\right)+G\left(u_{1,11}+u_{1,22}\right)+\frac{J}{4}\left(\ddot{u}_{1,22}-\ddot{u}_{2,12}\right) \\
& +\frac{1}{2} G \ell^{2}\left(u_{2,1222}+u_{2,1112}-u_{1,1122}-u_{1,2222}\right)=\rho \ddot{u}_{1}, \\
(\Lambda+G)\left(u_{1,12}+\right. & \left.u_{2,22}\right)+G\left(u_{2,11}+u_{2,22}\right)+\frac{J}{4}\left(\ddot{u}_{2,11}-\ddot{u}_{1,12}\right) \\
& +\frac{1}{2} G \ell^{2}\left(u_{1,1222}-u_{2,1122}+u_{1,1112}-u_{2,1111}\right)=\rho \ddot{u}_{2} .
\end{aligned}
$$

78 In vector form, the governing equation of plane strain elastodynamics of couple 79 stress bodies with micro-inertia reads

$(\Lambda+G) \operatorname{grad}_{2}\left(\operatorname{div}_{2} \boldsymbol{u}\right)+G \hat{\triangle} \boldsymbol{u}+\frac{J}{4} \operatorname{curl}_{2}\left(\operatorname{curl}_{2} \ddot{\boldsymbol{u}}\right)-\frac{1}{2} G \ell^{2} \hat{\triangle}\left[\operatorname{curl}_{2}\left(\operatorname{curl}_{2} \boldsymbol{u}\right)\right]=\rho \ddot{\boldsymbol{u}}$

$$
(\Lambda+2 G) \operatorname{grad}_{2}\left(\operatorname{div}_{2} \boldsymbol{u}\right)+\frac{J}{4} \operatorname{curl}_{2}\left(\operatorname{curl}_{2} \ddot{\boldsymbol{u}}\right)+G\left[1-\frac{1}{2} \ell^{2} \hat{\triangle}\right] \operatorname{curl}_{2}\left(\operatorname{curl}_{2} \boldsymbol{u}\right)=\rho \ddot{\boldsymbol{u}}
$$

87 which, again in the absence of micro-inertia, reduces to Eq.(141) of Hadjesfan-

88 diari and Dargush (2011) (provided that our $\ell$ is replaced by $\sqrt{2} \ell$ and that we 89 understand $\nabla \times \nabla \times \boldsymbol{u}$ as $\left.-\operatorname{curl}_{2} \operatorname{curl}_{2} \boldsymbol{u}=-\epsilon_{\alpha \beta} \epsilon_{\gamma \delta} u_{\delta, \gamma \beta}\right)$. 


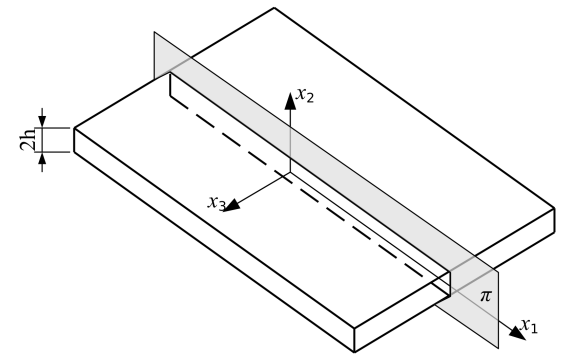

Figure 1: Plate in plane strain setting: the co-ordinate plane $\left(x_{1}, x_{3}\right)$ is the plate mid-plane

$$
\boldsymbol{q}=\boldsymbol{\mu}^{T} \boldsymbol{n}-\mu_{n n} \boldsymbol{n} .
$$

We now consider a flat plate in plane strain, see Fig/1. At the top/bottom face of the plate, $x_{2}= \pm h$, the unit normal vector is $\boldsymbol{n}=(0, \pm 1,0)$ such that, by referring to Eqs. 100 , we have $\left(\mu_{n n}=0\right)$

$$
\begin{aligned}
& p_{1}= \pm t_{21}= \pm\left(\sigma_{21}+\tau_{21}\right), \\
& p_{2}= \pm t_{22}= \pm \sigma_{22}, \\
& q_{3}= \pm \mu_{23} .
\end{aligned}
$$

96 2.2. Potentials

97

$98 \phi\left(x_{1}, x_{2}, x_{3}, t\right)$ and the vector potential $\boldsymbol{H}\left(x_{1}, x_{2}, x_{3}, t\right)$, such that (Graff and

99 Pao, 1967, Eq.(13)) 
In particular, under plane strain conditions (3), we have

$$
u_{1}=\frac{\partial \phi}{\partial x_{1}}+\frac{\partial H}{\partial x_{2}}, \quad u_{2}=\frac{\partial \phi}{\partial x_{2}}-\frac{\partial H}{\partial x_{1}},
$$

where we have let the shorthand $H=H_{3}\left(x_{1}, x_{2}, t\right)$, given that the other pair of components of $\boldsymbol{H}$ vanish. We observe that the gauge condition $\operatorname{div} \boldsymbol{H}=0$ is trivially satisfied. By substituting Eqs. (13) in (8) we obtain

$$
\begin{aligned}
G\left(1-\frac{1}{2} \ell^{2} \hat{\triangle}\right) \hat{\triangle} H & =\rho \ddot{H}-\frac{J}{4} \hat{\triangle} \ddot{H}, \\
(2 G+\Lambda) \hat{\triangle} \phi & =\rho \ddot{\phi},
\end{aligned}
$$

which, in the absence of micro-inertia, correspond to Eqs.(3) of Sengupta and Ghosh (1974) and Eqs.(8) of Wang et al. (2017).

Boundary conditions (11) may now be rewritten as

$$
\begin{aligned}
& p_{1}= \pm\left[G\left(H_{, 22}+2 \phi_{, 12}-H_{, 11}\right)+\frac{J}{4} \hat{\triangle} \ddot{H}-\frac{1}{2} G \ell^{2} \hat{\triangle}^{2} H\right] \\
& p_{2}= \pm\left[\Lambda \hat{\triangle} \phi+2 G\left(\phi_{, 22}-H_{, 12}\right)\right], \\
& q_{3}=\mp G \ell^{2} \hat{\triangle} H_{, 2},
\end{aligned}
$$

where $\hat{\triangle}^{2}=\nabla^{4}$ indicates the bi-harmonic operator.

\section{Travelling wave solutions}

In order to develop dimensional-reduced models, we need a good understanding of wave propagation in a thin plate. We begin by looking at solutions in the form of travelling waves

$$
\begin{aligned}
\phi\left(x_{1}, x_{2}\right) & =\Phi\left(x_{2}\right) \exp \left[\imath\left(k x_{1}-\omega t\right)\right], \\
H\left(x_{1}, x_{2}\right) & =\mathcal{H}\left(x_{2}\right) \exp \left[\imath\left(k x_{1}-\omega t\right)\right] .
\end{aligned}
$$

These, plugged into Eqs. 14, lends a pair of ODEs for the amplitude functions $\Phi\left(x_{2}\right)$ and $\mathcal{H}\left(x_{2}\right)=\mathcal{H}_{1}\left(x_{2}\right)+\mathcal{H}_{2}\left(x_{2}\right)$

$$
\Phi^{\prime \prime}-\frac{1}{\ell^{2}} \lambda_{L}^{2} \Phi=0, \quad \mathcal{H}_{1}^{\prime \prime}-\frac{1}{\ell^{2}} \lambda_{z 1}^{2} \mathcal{H}_{1}=0, \quad \mathcal{H}_{2}^{\prime \prime}-\frac{1}{\ell^{2}} \lambda_{z 2}^{2} \mathcal{H}_{2}=0,
$$

whereupon $\ell^{-1} \lambda_{L}$ and $\ell^{-1} \lambda_{z 1,2}$ are wavenumbers in the $x_{2}$ direction. 
We let the dimensionless frequency $\Omega=\omega T$, where $T=\ell / \tilde{c}_{S}$ is a reference time and $\tilde{c}_{S}=\sqrt{G / \rho}$ is the classical shear wavespeed. Also, we let the dimensionless wavenumber $\kappa=k \ell$, the rotational inertia characteristic length $\hbar=\frac{1}{2} \sqrt{J / \rho}$, as well as the dimensionless counterpart

$$
\mathfrak{J}=\hbar / \ell .
$$

With this, it is

$$
\lambda_{L}=\sqrt{\kappa^{2}-\theta_{L}^{2}}, \quad \theta_{L}^{2}=\Omega^{2} / \psi^{2},
$$

and

$$
\lambda_{z 1}=\sqrt{\kappa^{2}-\theta_{1}^{2}}, \quad \lambda_{z 2}=\sqrt{\kappa^{2}+\theta_{2}^{2}},
$$

where

$$
\theta_{1,2}^{2}=\sqrt{\left(\mathfrak{J}^{2} \Omega^{2}-1\right)^{2}+2 \Omega^{2}} \pm\left(\mathfrak{J}^{2} \Omega^{2}-1\right) \geq 0 .
$$

$\pm\left(\theta_{L}, \theta_{1}, \imath \theta_{2}\right)$ define the branch points of the square roots. In the absence of rotational inertia, $\theta_{1,2}$ reduce to $\sqrt{2} \ell(p, q)$ of Sengupta and Ghosh (1974) and to $\sqrt{2} \ell \beta_{1,2}$ of Graff and Pao (1967), respectively (just substitute our $\ell$ with $\sqrt{2} \ell$ ).

For $\kappa=0$, we get the so-called thickness-stretch or thickness-shear resonance frequencies (see Fig.5), with real wavenumber $\theta_{L}$ and $\theta_{1}$. We also have the non-classical evanescent thickness mode with wavenumber $\imath \theta_{2}$ (for a definition of evanescent modes see (Graff, 2012, §1.5.4)).

We observe that the classical limit is obtained taking $\ell \rightarrow 0$ and, if necessary, $J=0$. This limit, in terms of $\Omega$ and $\kappa$, amounts to taking $\Omega \sim \kappa \ll 1$, i.e. it is the long-wave low-frequency (LWLF) approximation. Within this approximation, we get

$$
\begin{aligned}
& \theta_{1}=\Omega-\frac{1}{2}\left(\frac{1}{2}-\mathfrak{J}^{2}\right) \Omega^{3}+\ldots \\
& \theta_{2}=\sqrt{2}+\frac{1}{\sqrt{2}}\left(\frac{1}{2}-\mathfrak{J}^{2}\right) \Omega^{2}+\ldots
\end{aligned}
$$

whence it is clear that, comparing with the classical results, for instance in the notation of (Graff, 2012, §8), we have

$$
\lambda_{L}^{2} \rightarrow-\ell^{2} \alpha^{2}, \quad \lambda_{1}^{2} \rightarrow-\ell^{2} \beta^{2}, \quad \lambda_{2}^{2} \rightarrow 2 .
$$



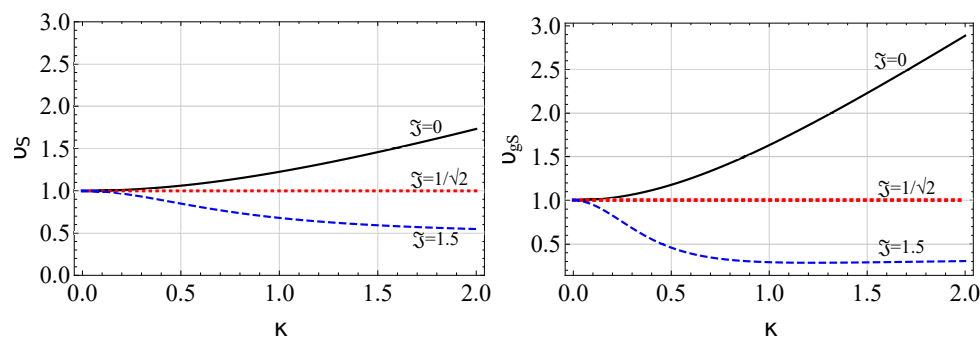

Figure 2: Phase (left) and group (right) speed of shear bulk waves, respectively Eq. 25 and 26 , normalized over the classical wave speed, $\tilde{c}_{S}$. Propagation is regular inasmuch as $\mathfrak{J}>\mathfrak{J}_{\text {crit }}=1 / \sqrt{2}$

133

$\mathcal{H}\left(\xi_{2}\right)=\frac{B_{1}}{\lambda_{z 1}} \sinh \left(\lambda_{z 1} \xi_{2}\right)+B_{2} \cosh \left(\lambda_{z 1} \xi_{2}\right)+\frac{C_{1}}{\lambda_{z 2}} \sinh \left(\lambda_{z 2} \xi_{2}\right)+C_{2} \cosh \left(\lambda_{z 2} \xi_{2}\right)$,

134 where we have let the dimensionless transversal coordinate $\xi_{2}=x_{2} / \ell$.

135

136 137

This limit corresponds to Case I of Sengupta and Ghosh (1974), although there it is claimed that the wavelength $L=2 \pi / k$ is large compared to $h$, while it really should be $L \gg \ell$.

We let the ratio between the longitudinal and the transversal wavespeed in classical media $\psi=\tilde{c}_{L} / \tilde{c}_{S}$, with $\tilde{c}_{L}=\sqrt{(2 G+\Lambda) / \rho}$. It is important to observe that $\theta_{1}>\theta_{L}$ demands

$$
0<\Omega<\psi \sqrt{\frac{\psi^{2}-1}{\frac{1}{2}-\mathfrak{J}^{2} \psi^{2}}},
$$

provided

$$
\mathfrak{J}<\frac{1}{\sqrt{2} \psi}
$$

otherwise $\theta_{1}>\theta_{L}$ always.

The general solutions of Eqs. 160 are

$$
\Phi\left(\xi_{2}\right)=\frac{A_{1}}{\lambda_{L}} \sinh \left(\lambda_{L} \xi_{2}\right)+A_{2} \cosh \left(\lambda_{L} \xi_{2}\right),
$$

3.1. Bulk waves

Bulk waves are travelling homogeneous waves, whence we get the dimensionless speed of longitudinal waves setting $\lambda_{L}=0$

$$
v_{L}=\psi,
$$


which is obviously non-dispersive. Similarly, we get the wavespeed of shear waves

$$
v_{S}=\sqrt{1+\frac{\frac{1}{2}-\mathfrak{J}^{2}}{1+\mathfrak{J}^{2} \kappa^{2}} \kappa^{2}},
$$

that generalizes the result (Ottosen et al. 2000, Eq.(33)), obtained in the absence of micro-inertia. Depending on the latter, two regimes are possible: when $\mathfrak{J} \lessgtr \mathfrak{J}_{\text {crit }} \equiv 2^{-1 / 2}$, the shear bulk speed is greater/lesser than the classical bulk wave speed $\tilde{c}_{S}$, while the particular case $\mathfrak{J}=\mathfrak{J}_{\text {crit }}$ corresponds to non-dispersive waves. This behaviour has already been observed for antiplane shear waves Nobili et al. (2019). The bulk shear wave speed asymptotes to

$$
v_{\text {Slim }}=\frac{\mathfrak{J}_{\text {crit }}}{\mathfrak{J}}
$$

which may be greater than the longitudinal wave speed $v_{L}=\psi$ if 22 holds. We conclude that shear waves are generally slower than bulk waves, unless condition (22) holds, in which case they still are but only in the frequency range expressed by (21). Therefore, the case where micro-inertia disappears is very special, in that shear waves are always dispersive and they eventually become faster than longitudinal waves, see Fig 2 .

Looking at group velocity

$$
V_{g S}=v_{S}+\frac{\mathfrak{J}_{\text {crit }}^{2}-\mathfrak{J}^{2}}{v_{S}\left(1+\mathfrak{J}^{2} \kappa^{2}\right)^{2}} \kappa^{2},
$$

we see that it is always positive. Besides, propagation is regular (i.e. group velocity is less than phase speed) inasmuch as $\mathfrak{J}>\mathfrak{J}_{\text {crit }}$, see Fig 2 , The corresponding dimensional speeds easily follow through

$$
c=\frac{\omega}{k}=\frac{\Omega}{\kappa} \tilde{c}_{S}=v \tilde{c}_{S}
$$

whereupon we see that the phase speed of longitudinal waves $c_{L}$ corresponds to the classical result $\tilde{c}_{L}$. In the absence of micro-inertia, longitudinal (24) 5 and transversal 25 wave speeds match the corresponding results (Sharma and Kumar, 2014, Eq.(7)). Dimensionalizing factors have been chosen such that in the classical limit, that is for $J$ and $\ell$ tending to zero, we rightly get $v_{S} \rightarrow 1$. 
together with the Rayleigh function

$$
R(\kappa, \Omega)=\frac{d_{0} \lambda_{L} \lambda_{z 1} \lambda_{z 2}+d_{1} \lambda_{z 2}-d_{2} \lambda_{z 1}}{\lambda_{z 1}-\lambda_{z 2}} .
$$

This function should be compared with Eq.(75) Graff and Pao (1967) of which it is a generalization in that (i) it encompasses for micro-inertia and (ii) it extracts the factor $\lambda_{z 1}-\lambda_{z 2}$ which cancels out spurious branch-cuts. The Rayleigh function may be rewritten as

$$
R_{0}(\kappa, \Omega)=\left(\Omega^{2}-2 \kappa^{2}\right)^{2}\left[1-\frac{\theta_{1}^{2}}{\lambda_{z 2}\left(\lambda_{z 1}+\lambda_{z 2}\right)}\right]-4 \kappa^{2} \lambda_{L} \lambda_{z 1} .
$$
which, in the limit as $\ell$ goes to zero (and letting $J=0$ ), corresponds, at leading 168 order, to the classical Rayleigh function. Indeed, we have $\kappa \sim \Omega \rightarrow 0$ and

$$
R_{0}(\kappa, \Omega)=\left(\lambda_{z 1}^{2}+\kappa^{2}\right)^{2}-4 \kappa^{2} \lambda_{L} \lambda_{z 1}+O\left(\Omega^{6}\right) .
$$




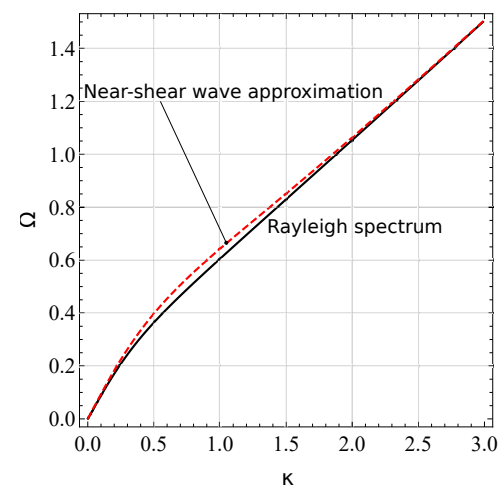

Figure 3: Frequency spectrum of Rayleigh waves (solid, black) superposed onto the approximation 28 (dashed, red) for the parameter set $\psi=1.5, \mathfrak{J}=1.5$

Rayleigh wave are perturbations of bulk shear waves and, as such, they may be sought in their close neighbourhood: for $\theta_{1}>\theta_{L}$ we have

$$
\kappa_{R}^{2}=\theta_{1}^{2}+\frac{\left(\theta_{1}^{2}+\theta_{2}^{2}\right) \theta_{2}^{4}\left(\Omega^{2}-2 \theta_{1}^{2}\right)^{4}}{\theta_{1}^{4}\left(4 \theta_{1}^{4}-4 \theta_{1}^{2}\left(S+\Omega^{2}\right)-4 \theta_{2}^{2} S+\Omega^{4}\right)^{2}},
$$

where we let the shorthand $S=\sqrt{\left(\theta_{1}^{2}+\theta_{2}^{2}\right)\left(\theta_{1}^{2}-\theta_{L}^{2}\right)}$. The quality of this approximation is excellent at both ends of the wavelength spectrum (i.e. for both short and long waves), as illustrated in Fig 3

\section{Rayleigh-Lamb waves}

For Rayleigh-Lamb (R-L) waves, we now impose free boundary conditions

$$
p_{1}\left(x_{1}, \pm h, t\right)=p_{2}\left(x_{1}, \pm h, t\right)=q_{3}\left(x_{1}, \pm h, t\right) \equiv 0 .
$$

where $h$ is the plate half-thickness, and consider symmetric and anti-symmetric waves separately.

\subsection{Symmetric waves}

For symmetric waves we obtain the frequency equation

$$
D_{s}=d_{0} \lambda_{L} \tanh \left(H \lambda_{L}\right)+\frac{d_{1}}{\lambda_{z 1}} \tanh \left(H \lambda_{z 1}\right)-\frac{d_{2}}{\lambda_{z 2}} \tanh \left(H \lambda_{z 2}\right),
$$

being $H=h / \ell$. It is straightforward matter to recognize that, in either the short-wave (SW) or high-frequency (HF) limit, respectively $\kappa \gg 1$ or $\Omega \gg 1$, 


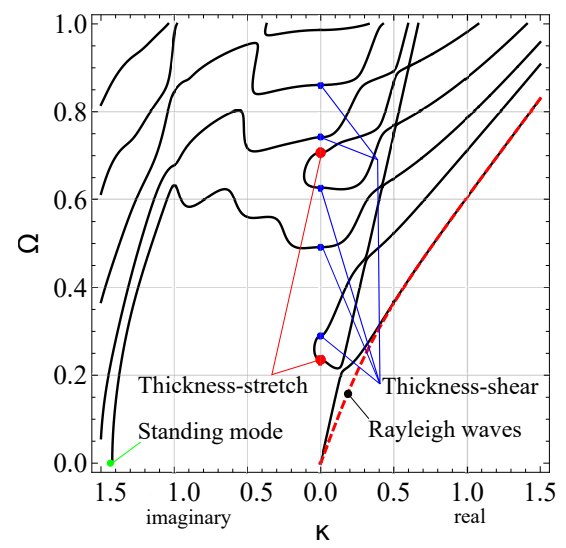

Figure 4: Frequency spectrum of symmetric Rayleigh-Lamb waves (black, solid) and Rayleigh waves (red, dashed) with the parameters $\psi=1.5, \mathfrak{J}=1.5$ and $H=10$. Clearly, the fundamental mode quickly asymptotes to Rayleigh waves. Thickness-stretch (red, large dots), thickness-shear (blue, small dots) cut-offs and standing modes (green dots) are also shown.

$D_{s}$ reduces to the numerator of the Rayleigh function (27), whence the first branch in the spectrum asymptotes to the Rayleigh branch. In fact, in such limit, the phase speed of all branches tends to that of Rayleigh waves, and this is in contrast to $\mathrm{CE}$, where branches other than the first tend to the bulk shear wave speed. Similarly, in the classical limit (20), we have, at leading order,

$$
d_{0}=-8 \ell^{2} k^{2}, \quad d_{1}=2 \ell^{4}\left(2 k^{2}-\frac{\omega^{2}}{\tilde{c}_{S}^{2}}\right)^{2}=2 \ell^{4}\left(k^{2}-\beta^{2}\right)^{2}, \quad d_{2}=O\left(\ell^{6}\right),
$$

whence Eq. 29) reduces to the classical result (Graff, 2012, Eq.(8.1.54))

$$
8 \ell^{3} k^{2} \alpha \tan (h \alpha)+\frac{2 \ell^{3}\left(k^{2}-\beta^{2}\right)^{2}}{\beta} \tan (h \beta)+O\left(\ell^{6}\right)=0 .
$$

Eq. 29) does not correspond to (16) of Sengupta and Ghosh (1974) because the latter is obtained taking a linear approximation in $\ell$. However, it is observed that, owing to the scaling, $\ell$ never appears explicitly in Eq. 297 . This means that, within the natural scaling and in plane-strain conditions, the couple-stress problem becomes self-similar. The frequency spectrum for symmetric waves is drawn in Fig, 4

Cut-on frequencies (below which the wave is evanescent and no longer prop- 


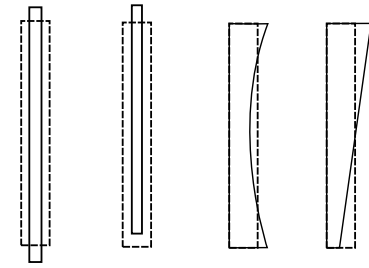
(a)
(b)
(c)
(d)

Figure 5: Thickness modes: (a) symmetric thickness-stretch, (b) antisymmetric thicknessstretch, (c) symmetric thickness-shear and (d) antisymmetric thickness-shear

194

agate Bhaskar (2009) ) are determined setting $\kappa=0$ in 29 , which lends

$$
\cos \left(H \theta_{L}\right)\left[\frac{\theta_{2}^{3} \tan \left(H \theta_{1}\right)}{\theta_{1}^{3} \tanh \left(H \theta_{2}\right)}+1\right]=0 .
$$

The presence of two factors is a consequence of the block structure of the linear system. Consequently, there are two families of modes (Graff, 2012, §8.7.5):

1. thickness-stretch modes, related to the first factor in (30),

$$
\Omega_{s n}=\left(\frac{1}{2}+n\right) \pi \frac{\psi}{H}, \quad n=0,1,2, \ldots,
$$

which are purely classical, cf. Kaplunov et al. 1998, Eq.(1.2.22)) and (Graff, 2012, Eq.(8.1.99)). For these modes, $A_{2}$ is arbitrary and $B_{1}=$ $C_{1}=0$, i.e. the displacement is irrotational. In this case, $u_{1} \equiv 0$ for $\kappa=0$ and indeed this is thickness-stretch, as in Fig 5 (a).

2. thickness-shear modes, which are non-classical. For such modes, $A_{2}=0$, whence $\phi \equiv 0$ and the displacement field is solenoidal according to the decomposition 12 , with rotational potential

$$
\mathcal{H}\left(\xi_{2}\right)=\left(\frac{\lambda_{z 2}^{2}}{\lambda_{z 1}} \frac{\sinh \left(\lambda_{z 1} \xi_{2}\right)}{\cosh \left(\lambda_{z 1} H\right)}-\frac{\lambda_{z 1}^{2}}{\lambda_{z 2}} \frac{\sinh \left(\lambda_{z 2} \xi_{2}\right)}{\cosh \left(\lambda_{z 2} H\right)}\right) R
$$

where $R$ is a free parameter. Displacement easily follows from Eqs.13), which give $u_{2} \equiv 0$ at cut-off (because $\kappa=0$ ) and confirm that this is indeed thickness-shear, as in Fig.5(c)

We observe that, for both thickness mode families, cut-on frequencies are $O\left(H^{-1}\right)$. Standing modes, i.e. wave solutions for which $\Omega=0$, are given by either $\kappa=$ 0 , corresponding to rigid body motions, or by the solutions of the transcendental 


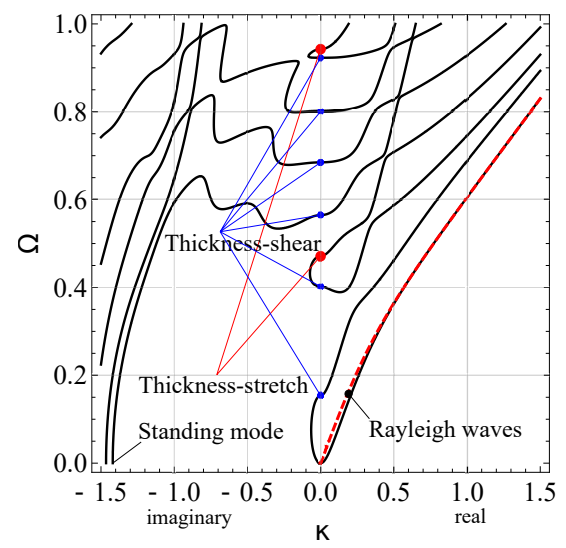

Figure 6: Frequency spectrum of antisymmetric (flexural) Rayleigh-Lamb waves (black, solid) superposed onto Rayleigh waves (red, dashed). The fundamental mode quickly asymptotes to Rayleigh waves. Antisymmetric thickness-shear (blue, small dots) and thickness-stretch (red, big dots) are shown, for the parameter set $\psi=1.5, \mathfrak{J}=1.5$ and $H=10$.

equation in $k_{2}=\Im(k)$

$$
\begin{aligned}
& 2 H k_{2}\left(\psi^{2}-1\right)-\left(\left(k_{2}^{2}-1\right) \psi^{2}+1\right) \sin \left(2 H k_{2}\right) \\
& +\frac{2 k_{2}^{3} \psi^{2}}{\sqrt{2-k_{2}^{2}}} \cos ^{2}\left(H k_{2}\right) \tanh \left(H \sqrt{2-k_{2}^{2}}\right)=0 .
\end{aligned}
$$

Assuming $H^{-1} \ll 1$, these may be found as perturbations of classical standing modes

$$
\kappa= \pm \imath \sqrt{2+\left(\frac{1}{2}+j\right)^{2} \frac{\pi^{2}}{H^{2}}}+O\left(H^{-2}\right), \quad j=0,1,2, \ldots
$$

Standing modes are associated with a displacement field where $C_{1}=0$ and $A_{2}$ is linearly proportional to $B_{1}$. Unlike cut-ons, standing modes are $O(1)$ even when $H$ is large and cannot be captured in a long-wave approximation.

\subsection{Antisymmetric waves}

Similarly, for anti-symmetric waves, we get

$$
D_{o}=d_{0} \lambda_{L} \operatorname{coth}\left(H \lambda_{L}\right)+\frac{d_{1}}{\lambda_{z 1}} \operatorname{coth}\left(H \lambda_{\mathrm{z} 1}\right)-\frac{d_{2}}{\lambda_{z 2}} \operatorname{coth}\left(H \lambda_{\mathrm{z} 2}\right),
$$

whose first branch, once again, collapses into Rayleigh waves (27) in the shortwave regime, see Fig 6 Proceeding as for symmetric waves, it is easily shown 
that, in the classical limit, the frequency equation 32 reduces to the well-known result

$$
-8 \ell^{3} k^{2} \alpha \tan (h \alpha)+\frac{2 \ell^{3}\left(k^{2}-\beta^{2}\right)^{2}}{\beta} \tan (h \beta)+O\left(\ell^{6}\right)=0 .
$$

Cut-on frequencies are given by

$$
\sin \left(H \theta_{L}\right)\left[\frac{\theta_{2}^{3} \cot \left(H \theta_{1}\right)}{\theta_{1}^{3} \operatorname{coth}\left(H \theta_{2}\right)}-1\right]=0,
$$

whence, again, two families of modes arise

1. antisymmetric thickness-stretch modes, purely classical, with frequency (Kaplunov et al., 1998, Eq.(1.2.25))

$$
\Omega_{o n}=n \pi \frac{\psi}{H}, \quad n=0,1,2, \ldots
$$

for which $A_{1}$ is arbitrary and $B_{2}=C_{2}=0$, i.e. the displacement is irrotational. The last feature accounts for the fact that $u_{1} \equiv 0$ at cut-off and this is thickness-stretch, as in Fig 5 (b).

2. antisymmetric thickness-shear modes, non-classical, for which $A_{1}=0$ and the displacement field is solenoidal, with potential

$$
\mathcal{H}\left(\xi_{2}\right)=\left(\frac{\lambda_{z 2}^{3}}{\lambda_{z 1}} \frac{\cosh \left(\lambda_{z 1} \xi_{2}\right)}{\sinh \left(\lambda_{z 1} H\right)}-\frac{\lambda_{z 1}^{3}}{\lambda_{z 2}} \frac{\cosh \left(\lambda_{z 2} \xi_{2}\right)}{\sinh \left(\lambda_{z 2} H\right)}\right) R,
$$

where $R$ is a free parameter. For a solenoidal field, only $u_{1}$ can be nonzero at cut-off, and again we have thickness-shear, although this time in antisymmetric fashion, as in Fig/5(d).

Standing modes are given by

$$
\kappa= \pm \imath \sqrt{2+n^{2} \frac{\pi^{2}}{H^{2}}}, \quad n=1,2, \ldots,
$$

and are non-classical. They are associated with eigenforms where $B_{2}, C_{2}$ are linear functions of $A_{1}$.

\section{Construction of the asymptotic models}

Asymptotic models are constructed on the assumption that the wavelength of interest is much greater than the microstructural parameter $\ell$, i.e. $\kappa \ll 1$, and 


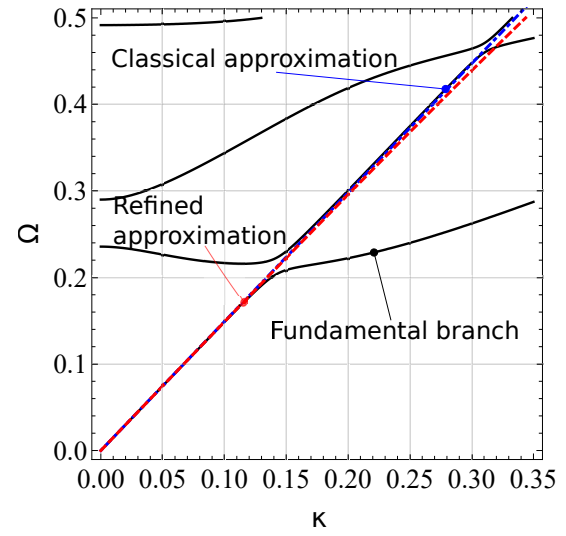

Figure 7: Frequency spectrum of symmetric Rayleigh-Lamb waves superposed onto the leading order (blue, dot-dashed) and first correction approximation (red, dashed) for the parameter set $\psi=1.5, \mathfrak{J}=1.5$ and $H=10$.

237

that the time scale of wave propagation is much greater than $T$, i.e. $\Omega \ll 1$. We emphasize that this not necessarily corresponds to the classical limit, whereby $\ell \rightarrow 0$. In the following, unless otherwise stated, we assume $H \sim 1$.

\subsection{Extensional plate model}

Looking at the frequency spectrum of the symmetric fundamental mode, we see that $\Omega \sim \kappa$ and we write the LWLF approximation

$$
D_{s}=\Omega^{2}-4\left(1-\psi^{-2}\right) \kappa^{2}+O\left(\kappa^{4}\right)
$$

corresponding to classical longitudinal waves in a plate

$$
\square_{P 1} u=c_{P 1}^{-2} \frac{\partial^{2} u}{\partial t^{2}}-\frac{\partial^{2} u}{\partial x_{1}^{2}}=0,
$$

36 being $\square_{P 1}$ the wave (or D'Alambert's) operator with wave speed $c_{P 1}$ (sometimes denoted $c_{3}$ )

$$
c_{P 1}^{2}=4 \tilde{c}_{S}^{2}\left(1-\psi^{-2}\right)=\frac{E}{\rho\left(1-\nu^{2}\right)}=\frac{2}{1+\nu} \tilde{c}_{S}^{2},
$$

where $E=G(2 G+3 \Lambda) /(G+\Lambda)$ is Young's modulus and $\nu=\frac{1}{2} \Lambda /(\Lambda+G)$ Poisson's ratio. The wave phase speed $c_{P 1}>\tilde{c}_{S}$ corresponds to that of classical plate theory, see (Graff] 2012, Eq.(8.3.57)), and indeed Eq.35) is the plane-strain counterpart of the classical equation of extensional vibrations of thin plates, 
originally developed by Poisson (1829), Cauchy (1828) and Filon (Mindlin and Yang, 2006, Eq.(4.043))

$$
\square_{P 1} u=\frac{1-\nu^{2}}{2 h E} b_{1},
$$

where $b_{1}$ is the longitudinal force acting on the plate. This linear approximation (of the dispersion relation) is very robust (see Fig77) and classical, for it is independent of $\ell, H$ and $\mathfrak{J}$. As it might be expected, rotational inertia appears when accounting for the first order correction terms in the asymptotic expansion of the frequency equation. Indeed, we have

$$
\begin{aligned}
D_{s}=\Omega^{2} & -4\left(1-\psi^{-2}\right) \kappa^{2} \\
& +\frac{\left(1-2 \psi^{-2}\right)^{2}}{4\left(1-\psi^{-2}\right)}\left[\frac{4}{3}\left(1-\psi^{-2}\right) H^{2}+\frac{\sqrt{2}}{H} \tanh (\sqrt{2} H)-2\right] \Omega^{2} \kappa^{2}+\ldots
\end{aligned}
$$

whence we obtain the asymptotic model

$$
\frac{E A}{1-\nu^{2}} \frac{\partial^{2} u}{\partial x_{1}^{2}}-\mathfrak{I}_{\mathrm{tg}} u=0,
$$

where we have defined the operator of modified tangential inertia in analogy with (Kaplunov et al. $1998, \S 7)$

$$
\mathfrak{I}_{\operatorname{tg}}=\rho A\left\{1-\frac{\nu^{2}}{2(1-\nu)}\left[\frac{2}{3(1-\nu)} h^{2}-\ell^{2}\left(2-\sqrt{2} \frac{\ell}{h} \tanh \frac{\sqrt{2} h}{\ell}\right)\right] \frac{\partial^{2}}{\partial x_{1}^{2}}\right\} \frac{\partial^{2}}{\partial t^{2}} .
$$

239 Remarkably, the contribution of the microstructure affects plate elongation by reducing rotational inertia (that is the term with the mixed derivative), independently of $J$. Indeed, the term in round bracket is always positive and ranges from 0 to 2 , respectively at $H=0$ and as $H \rightarrow \infty$. As a result, the fundamental spectrum decays less than in the classical situation.

In the classical limit $\ell \rightarrow 0$, we get

$$
\frac{E A}{1-\nu^{2}} \frac{\partial^{2} u}{\partial x_{1}^{2}}-I_{\mathrm{tg}} u=0,
$$

244

where $A=2 h$ is the transversal thickness of the plate and

$$
I_{\mathrm{tg}}=\rho A\left(1-h^{2} \frac{\nu^{2}}{3(1-\nu)^{2}} \frac{\partial^{2}}{\partial x_{1}^{2}}\right) \frac{\partial^{2}}{\partial t^{2}},
$$




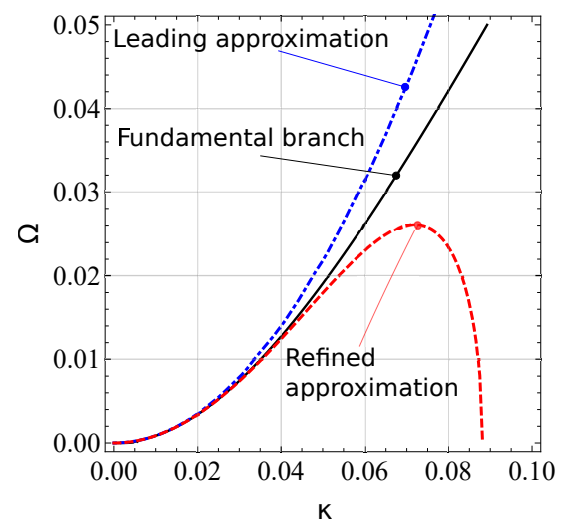

Figure 8: Frequency spectrum of antisymmetric (flexural) Rayleigh-Lamb waves superposed onto the leading order (green, dashed) and first correction approximation (red, dotted) for the parameter set $\psi=1.5, \mathfrak{J}=1.5$ and $H=10$.

corresponds to the result given in (Kaplunov et al., 1998, Eq.(7.4.3)) in the context of classical elasticity.

The asymptotically consistent result (36) differs from any engineering theory of plate extensional vibrations. For example, by analogy with (Kaplunov et al. 1998, Eq.(7.4.3)), we can easily generalize our result outside plane strain

$$
\frac{E A}{1-\nu^{2}}\left(\frac{1}{1+\nu} \hat{\triangle}+\frac{1}{1-\nu} \operatorname{grad}_{2} \operatorname{div}_{2}\right) \boldsymbol{u}-\mathfrak{I}_{\mathrm{tg}} \boldsymbol{u}=\boldsymbol{o},
$$

and this equation is in disagreement with Eqs.(16a,b) of Jomehzadeh et al. (2011). In fact, they differ by the very way microstructure operates: by affectInterestingly, models agree to leading order under plane strain.

We now consider the limit $H \rightarrow 0$ of a plate whose thickness is much smaller than the microstructural length $\ell$. Then, we have

$$
\frac{E A}{1-\nu^{2}} \frac{\partial^{2} u}{\partial x_{1}^{2}}-\rho A\left\{1+\frac{\nu^{2}(1-2 \nu)}{3(1-\nu)^{2}} h^{2} \frac{\partial^{2}}{\partial x_{1}^{2}}\right\} \frac{\partial^{2} u}{\partial t^{2}}=0,
$$

and rotational inertia operates in the same direction as translational inertia, i.e. the spectrum grows. Remarkably, in this limit, $\ell$ drops out. 
As in the classical situation, we seek for a quadratic approximation for the fundamental flexural branch $\Omega \sim \kappa^{2} \ll 1$

$$
D_{o}=\Omega^{2}-2\left[1+\frac{2}{3} H^{2}\left(1-\psi^{-2}\right)\right] \kappa^{4}+O\left(\kappa^{6}\right),
$$

corresponding to dispersive waves in a flexural plate

$$
\mathfrak{L} w=\left[\rho A \frac{\partial^{2}}{\partial t^{2}}+\left(D^{\ell}+D\right) \frac{\partial^{4}}{\partial x_{1}^{4}}\right] w=0,
$$

which corresponds to the asymptotic model

$$
\left(D^{\ell}+D\right) \frac{\partial^{4} w}{\partial x_{1}^{4}}+\Im_{\operatorname{tr}} w=0 .
$$

where we have defined the operator of modified transverse inertia

$$
\begin{aligned}
\mathfrak{I}_{\mathrm{tr}}=\rho A\{1- & {\left[2 \ell^{2}+\frac{J}{\rho}+\frac{D^{\ell}}{D+D^{\ell}} \times\right.} \\
& \left.\left.\left(\frac{17-7 \nu}{45(1-\nu)^{2}} \frac{h^{4}}{\ell^{2}}-\ell^{2}\left(2-\frac{h}{\sqrt{2} \ell} \operatorname{coth} \frac{\sqrt{2} h}{\ell}\right)\right)\right] \frac{\partial^{2}}{\partial x_{1}^{2}}\right\} \frac{\partial^{2}}{\partial t^{2}} .
\end{aligned}
$$


Here, microstructure adds to rotational inertia directly through $2 \ell^{2}+J / \rho$, but it also contribute to/subtract from the classical term inasmuch as $H \gtrless 2.8265$. Eqs. (36) and (40) extend to microstructured plates the classical Kirchhoff-Love theory with modified inertia presented in Kaplunov et al. (1998). Indeed, in the limit as $\ell \rightarrow 0$ and setting $J=0$, we get

$$
D \frac{\partial^{4} w}{\partial x_{1}^{4}}+I_{\mathrm{tr}} w=0
$$

268

so by the factor $\frac{12}{5} \leq \frac{17-7 \nu}{5(1-\nu)} \leq \frac{27}{5}$ in the rotational inertia term. Even more 281 it disagrees with the engineering theory with size-dependence. For example,

$$
\left(D^{\ell}+D\right) \nabla^{4} w+\mathfrak{I}_{\operatorname{tr}} w=0
$$

where $\nabla^{4}$ is the bi-harmonic operator and $\mathfrak{I}_{\text {tr }}$ is generalized with the rotational inertia term $\hat{\triangle} \frac{\partial^{2}}{\partial t^{2}}$. Again, this result is at variance with the engineering theories. In particular, in the classical limit, it does not correspond to the classical Kirchhoff-Rayleigh theory of plates

$$
\left[D \nabla^{4}+\rho A\left(1-\frac{I_{2}}{A} \triangle\right) \frac{\partial^{2}}{\partial t^{2}}\right] w=0
$$




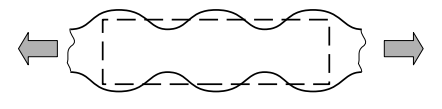

(a)

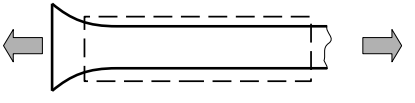

(b)

Figure 9: Thickness-stretch modes in a plate: real wavespeed (a) and evanescent mode for a semi-infinite domain (b)

according to (Jomehzadeh et al., 2011, Eq.(16c)), microstructure only affects the plate flexural rigidity and contributes nothing to rotational inertia, that is purely geometrical, just as in the classical case of Kirchhoff-Rayleigh plates. The same occurs, among many, in Ma et al. (2008) and in the Mindlin-like model Ma et al. (2011).

Finally, we consider the limit $H \rightarrow 0$, corresponding to the microstructuredominated regime. Then, we find

$$
D^{\ell} \frac{\partial^{4} w}{\partial x_{1}^{4}}+\rho A\left\{1-\left(\frac{1}{2} \ell^{2}+\frac{J}{\rho}\right) \frac{\partial^{2}}{\partial x_{1}^{2}}\right\} \frac{\partial^{2} w}{\partial t^{2}}=0,
$$

which is independent of $h$. It so appears that, in this limit, flexural and elongational waves depend in disjoint fashion on $\ell$ and $h$, respectively.

\section{High order modes}

We now consider the second branch of the spectrum (the first overtone) and its leading order approximation for $\Omega, \kappa \ll 1$. This limit, which is named longwave high-frequency (Kaplunov et al., 1998, §1.2), is capable of capturing the first cut-on frequency inasmuch as $\epsilon \sim \Omega \ll 1$ and, as it will be presently seen, $\epsilon=H^{-1}$ for elongation or $\epsilon=\sqrt{H^{2}+\frac{\pi^{2}}{4} \mathfrak{J}^{2}}$ for flexural vibrations.

\subsection{Symmetric waves}

For symmetric waves, we assume $\Omega \sim \kappa \sim \epsilon$ and find the expansion

$$
D_{2 s}=\Omega^{2}-\psi^{2} \varkappa_{s} \kappa^{2}-\Omega_{s 0}^{2},
$$

where $\Omega_{s 0}$ is the first cut-on frequency for symmetric waves (31) and refers to a thickness-stretch mode. In terms of the reduced model, this mode corresponds to squeezing and thickening of the cross-section, see Fig.9. The coefficient $\varkappa_{s}$ 
may be given in closed form, but its expression is cumbersome and it is not reported here. Instead, we write it in the form of an asymptotic expansion in $\epsilon$, namely $\varkappa_{s}=\varkappa_{s}^{(0)}+\epsilon^{2} \varkappa_{s}^{(2)}+\ldots$, and find

$$
\varkappa_{s}=1+\frac{16}{\pi \psi^{3}} \cot \left(\frac{1}{2} \pi \psi\right)+\ldots,
$$

which corresponds to (Kaplunov et al., 1998, Eq.(1.2.23)). As expected, microstructural effects only appear as first order correction terms. It is worth emphasizing that two regimes are possible: for $\varkappa_{s}^{(0)}>0, \Omega_{s 0}^{2}$ is really a cut-on and we have the wave equation

$$
\left[\square_{2 s}+\frac{\pi^{2}}{4 h^{2} \varkappa_{s}^{(0)}}\right] u=0
$$

where the wave operator $\square_{2 s}$ is connected to the real wave speed $c_{2 s}=\tilde{c}_{L} \sqrt{\varkappa_{s}^{(0)}}$. In this regime, the first branch describes a travelling thickness-stretch deformation mechanism as a tout string with the addition of pre-stress. In contrast, for $\varkappa_{s}^{(0)}<0$ (that first occurs in the range $1.21612<\psi<2$ ), $\Omega_{s 0}^{2}$ is locally a cut-off and we obtain a decaying (evanescent) mode, which is important for semi-infinite or finite domains, Fig 9 (b) Nobili et al. (2020). Thus, an asymptotically consistent theory accounting for the first two branches of symmetric waves is purely classical

$$
\left(\square_{2 s}+\frac{\pi^{2}}{4 h^{2} \varkappa_{s}^{(0)}}\right) \square_{P 1} u=0,
$$

to leading order in $\epsilon$.

When moving to first correction terms, it may be observed that the coefficient of $\kappa^{4}$ in the expansion of the frequency equation is $O\left(\epsilon^{2}\right)$ and therefore simply the correction term

$$
\varkappa_{s}^{(2)}=\frac{\pi}{\psi}\left[\pi\left(\frac{1}{2}-\mathfrak{J}^{2}\right) \psi+\left(\mathfrak{J}^{2}+\frac{1}{2}\right) \sin (\pi \psi)\right] \csc ^{2} \frac{\pi \psi}{2}
$$

needs to be considered in (43). The quality of this refined approximation is very good and, in Fig 10 , it is compared with the leading order approximation $\varkappa_{s}^{(0)}$ and the full coefficient $\varkappa_{s}$. It is most interesting to observe that there are special values for $\psi$, the first of which being $\psi=1.21612$, such that $\varkappa_{s}^{(0)}=0$ and the correction term 45 becomes leading order. 


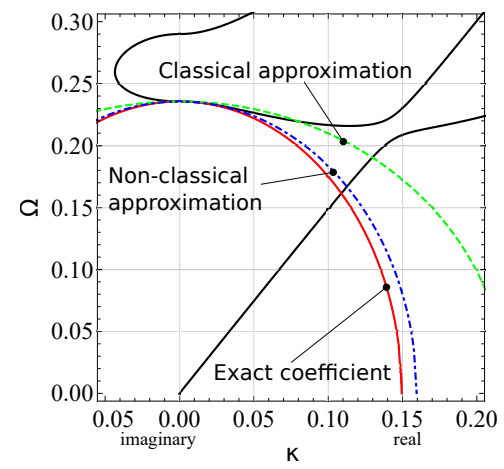

Figure 10: Frequency spectrum for symmetric R-L waves (solid, black) superposed onto the approximation 42 taking the leading order term 43 (green, dashed), the first correction approximation $\varkappa_{s}^{(0)}+\epsilon^{2} \varkappa_{s}^{(2)}$ (blue, dot-dashed) and the full coefficient $\varkappa_{s}$ (red, solid). It is clear that the first order approximation is very efficient. Here, $1.21612<\psi=1.5<2$ and we see spectrum decay, which means that this is an evanescent mode for the plate $(H=10, \mathfrak{J}=1.5)$

\subsection{Antisymmetric waves}

We now consider the second branch for antisymmetric waves, which is an antisymmetric thickness-shear mode. In terms of the cross-section of a plate model, this resembles shear-warping in the fashion considered by Timoshenko theories. Although now an explicit solution for the cut-on frequency equation (33) is not available, we can observe that, for $H=0$, we have the single solution

$\Omega_{o 0}=\mathfrak{J}^{-1}$, while for $H^{-1} \rightarrow 0$, we have the expansion

$$
\Omega_{o 0}=\frac{\pi}{2 H}\left(1+\frac{\frac{1}{2}-\mathfrak{J}^{2}}{8 H^{2}}+\ldots\right) .
$$

225 Consequently, to leading order, we adopt the composite expansion

$$
\Omega_{o 0}=\frac{\pi}{2 \sqrt{H^{2}+\frac{\pi^{2}}{4} \mathfrak{J}^{2}}}
$$

326 which proves extremely accurate for $\epsilon=\left(H^{2}+\frac{\pi^{2}}{4} \mathfrak{J}^{2}\right)^{-1 / 2}$ small, see Fig 11

27 Clearly, this expression for the first cut-on is non-classical. Thus, we assume

${ }_{28} \Omega \sim \epsilon$ and the dispersion relation takes the form

$$
D_{2 o}=\Omega^{2}-\frac{\pi^{2}}{4 H^{2}+\pi^{2} \mathfrak{J}^{2}}-\varkappa_{o} \kappa^{2} .
$$

For the coefficient $\varkappa_{0}$, an explicit expression is available but it is rather involved and we do not present it here. Instead, we observe that, in the 

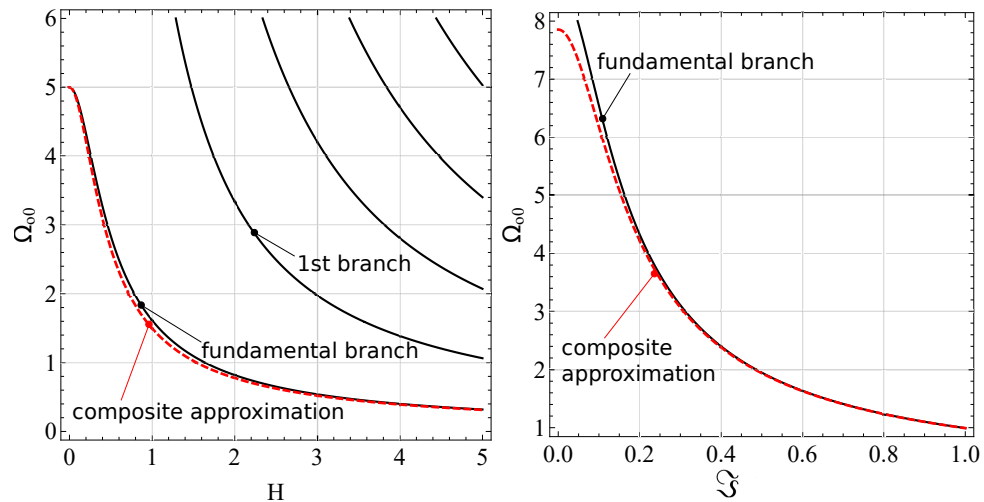

Figure 11: Cut-on frequencies for antisymmetric waves as a function of $H$ for $\mathfrak{J}=0.2$ (left) and as a function of $h$ for $H=0.2$ (right), superposed onto the composite expansion 46 for the first branch (red, dashed)
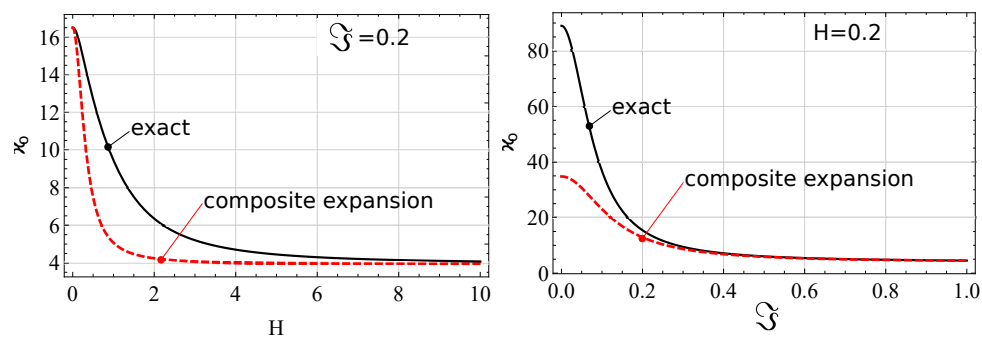

Figure 12: Coefficient $\varkappa_{0}$ as a function of $H$ for $\mathfrak{J}=0.2$ (left) and as a function of $\mathfrak{J}$ for $H=0.2$ (right), superposed onto the composite expansion 49) (red, dashed) 
331

microstructure-dominated regime,

$$
\varkappa_{o}=4+\frac{1}{2 \mathfrak{J}^{2}}+\frac{8 \mathfrak{J}^{2}\left(-\frac{2}{\psi^{2}}+\frac{60}{\pi^{2}}-5\right)+\frac{60}{\pi^{2}}-7}{12 \mathfrak{J}^{4}} H^{2}+\ldots, \quad H \ll 1,
$$

332

while, in the classical limit, we retrieve (Kaplunov et al. 1998, Eq.(1.2.27)

$$
\varkappa_{o}=1+\frac{16}{\pi \psi} \cot \frac{\pi}{2 \psi}+\ldots, \quad H \rightarrow \infty .
$$

${ }_{333}$ Hence, we introduce the composite expansion

$$
\varkappa_{o}=\frac{\left(1+\frac{16}{\pi \psi} \cot \frac{\pi}{2 \psi}\right) H^{2}+\frac{\pi^{2}}{8}\left(1+8 \mathfrak{J}^{2}\right)}{H^{2}+\frac{\pi^{2}}{4} \mathfrak{J}^{2}} \geq 0,
$$

which is $O(1)$ and achieves excellent accuracy for $\epsilon$ small, see Fig 12 Thus, we have $\kappa=O(\epsilon)$ and we disregard the closest neighbourhood of the cut-on frequency, that is to say that $\Omega-\Omega_{o 0} \sim \epsilon$, see Erbaş et al. (2018) for more details in the context of a Kirchhoff plates resting on a Winkler foundation. Therefore, we retrieve the wave equation

$$
\square_{o} w+\frac{\pi^{2}}{\left(4 h^{2}+\frac{\pi^{2}}{4} J / \rho\right) \varkappa_{o}} w=0,
$$

334 with real speed $c_{o}=\tilde{c}_{S} \sqrt{\varkappa_{o}}>\tilde{c}_{S}$.

In this equation, microstructure appears mostly (but not only) through micro-inertia. In the classical limit, this approximation becomes purely classical and it can be compared with the second branch of the Timoshenko theory. As observed in Bhaskar (2009), this second branch is able to capture the cut-on frequency upon letting $\varkappa_{T}=\pi^{2} / 12$, whence the requirement $\sigma \approx 4$ demands $\nu=0.644$, which is unacceptable. Besides, with this choice, Timoshenko second branch has the form (Bhaskar, 2009, Eq.(2.7))

$$
D_{2 o}=\Omega^{2}-\Omega_{o 0}^{2}\left(1+\frac{1+\sigma}{6} h^{2} \kappa^{2}\right)
$$

335

336

where $\Omega_{o 0}=\frac{\pi}{2 H}$. This expression is clearly missing the $O\left(\epsilon^{2}\right)$ term, in favour of the higher order contribution $\Omega_{o 0}^{2} \kappa^{2}$.

We conclude that an higher order theory accounting for the first two branches of flexural waves is given by

$$
\left(\square_{o}+\frac{\pi^{2}}{\left(4 h^{2}+\frac{\pi^{2}}{4} J / \rho\right) \varkappa_{0}}\right)\left[\left(D^{\ell}+D\right) \nabla^{4}+\rho A \frac{\partial^{2}}{\partial t^{2}}\right] w=0,
$$


to leading order in $\epsilon$.

\section{Conclusions}

We develop a new model for plate elongation and flexure, incorporating sizedependence, by asymptotic reduction of the elastodynamics of a thin plate made of elastic isotropic couple-stress material with micro-inertia. This model is consistent, in that it reproduces the dispersion features of the fundamental mode of the 3D body. It is also unique, it being only possible to refine the degree of approximation or the number of modes it captures. This is in contrast with the numerous models recently presented in the literature, which are obtained enforcing specific kinematical assumptions onto Hamilton's principle (engineering models). Consequently, these models may well differ in the elastic part, depending on the assumed kinematics, be it either Kirchhoff's or Mindlin's or many other's. Conversely, models are very similar in the inertia terms, in which microstructure usually does not appear. This is because it is hardly possible to anticipate the actual distribution of inertia forces. Asymptotic reduction shows that the opposite holds true, in that the classical solution is the leading order approximation, while microstructure appears, as the first order correction, in the form of rotational inertia terms. As a noticeable exception, couple stresses contribute at leading order to the flexural rigidity of the plate, and this is indeed a result that we share with the engineering models.

It is also noteworthy that asymptotic results are independent of the specific modification of the couple-stress theory that we may start from, namely reduced couple-stress or strain gradient effect. This shows that all couple-stress originated models are equivalent, when it comes to dimensional-reduced theories. Conversely, micro-inertia plays an important role in some fundamental features of the Rayleigh-Lamb spectrum, and it cannot be neglected.

When we turn to higher order models, we see that symmetric modes are fundamentally classical, and therefore microstructure appears, again, as a correction term. This is perhaps expected, since couple-stress theory provides results very similar to classical elasticity in terms of longitudinal waves. Conversely, 
microstructure already determines the leading term approximation for antisymmetric thickness-shear modes. The resulting size-dependent approximation can be intended to incorporate shear deformations, in the spirit of Timoshenko.

Finally, it is perhaps useful to compare the asymptotic approach with Mindlin's (Mindlin and Yang, 2006, Graff, 2012), which adopts Taylor expansions in the thickness direction (an idea originally proposed by Poisson, cf Love (1888)). Despite the two methods being vastly different, the latter still exploits consistency with the 3D body dispersion features to set tunable parameters (Graff. 2012. $\S 8.3 .1)$. We conclude that spectrum consistency is a transversal concept, which is not tied to the realm of asymptotic reduction.

\section{Acknowledgements}

The author acknowledges financial support from POR FESR 2014-2020 ASSE 1 AZIONE 1.2.2, Project IMPReSA, CUP E81F18000310009.

\section{Declaration of interest}

The author has no competing interest to declare.

\section{References}

Bhaskar, A., 2009. Elastic waves in Timoshenko beams: the lost and found of an eigenmode. Proceedings of the Royal Society A: Mathematical, Physical and Engineering Sciences 465, 239-255.

Chong, A., Yang, F., Lam, D.C., Tong, P., 2001. Torsion and bending of micronscaled structures. Journal of Materials Research 16, 1052-1058.

Erbaş, B., Kaplunov, J., Nobili, A., Kılıç, G., 2018. Dispersion of elastic waves in a layer interacting with a Winkler foundation. The Journal of the Acoustical Society of America 144, 2918-2925.

Eringen, A.C., 1984. Theory of nonlocal elasticity and some applications. Technical Report. PRINCETON UNIV NJ DEPT OF CIVIL ENGINEERING. 
Fleck, N., Hutchinson, J., 1993. A phenomenological theory for strain gradient effects in plasticity. Journal of the Mechanics and Physics of Solids 41, 18251857.

Graff, K., 2012. Wave motion in elastic solids. Dover Publications, Inc - New York.

Graff, K., Pao, Y.H., 1967. The effects of couple-stresses on the propagation and reflection of plane waves in an elastic half-space. Journal of Sound and Vibration 6, 217-229.

Gurtin, M., Murdoch, A., 1975. A continuum theory of elastic material surfaces. Archive for rational mechanics and analysis 57, 291-323.

Hadjesfandiari, A., Dargush, G., 2011. Couple stress theory for solids. International Journal of Solids and Structures 48, 2496-2510.

Jomehzadeh, E., Noori, H., Saidi, A., 2011. The size-dependent vibration analysis of micro-plates based on a modified couple stress theory. Physica E: Low-dimensional Systems and Nanostructures 43, 877-883.

Kaplunov, J., Kossovitch, L., Nolde, E., 1998. Dynamics of thin walled elastic bodies. Academic Press.

Kirchhoff, G., 1859. Ueber das gleichgewicht und die bewegung eines unendlich dünnen elastischen stabes. Journal für die reine und angewandte Mathematik $1859,285-313$.

Koiter, W., 1969. Couple-stresses in the theory of elasticity, i \& ii. Philosophical Transactions of the Royal Society of London B .

Lazopoulos, K., 2004. On the gradient strain elasticity theory of plates. European Journal of Mechanics-A/Solids 23, 843-852.

Lazopoulos, K., 2009. On bending of strain gradient elastic micro-plates. Mechanics Research Communications 36, 777-783. 
Love, A., 1888. Xvi. the small free vibrations and deformation of a thin elastic shell. Philosophical Transactions of the Royal Society of London.(A.) , 491546.

Ma, H., Gao, X.L., Reddy, J., 2008. A microstructure-dependent timoshenko beam model based on a modified couple stress theory. Journal of the Mechanics and Physics of Solids 56, 3379-3391.

Ma, H., Gao, X.L., Reddy, J., 2011. A non-classical Mindlin plate model based on a modified couple stress theory. Acta mechanica 220, 217-235.

Mikhasev, G., Nobili, A., 2020. On the solution of the purely nonlocal theory of beam elasticity as a limiting case of the two-phase theory. International Journal of Solids and Structures 190, 47-57.

Mindlin, R., Yang, J., 2006. An introduction to the mathematical theory of vibrations of elastic plates. World Scientific.

Nobili, A., Radi, E., Signorini, C., 2020. A new rayleigh-like wave in guided propagation of antiplane waves in couple stress materials. Proceedings of the Royal Society A 476, 20190822.

Nobili, A., Radi, E., Vellender, A., 2019. Diffraction of antiplane shear waves and stress concentration in a cracked couple stress elastic material with micro inertia. Journal of the Mechanics and Physics of Solids 124, 663-680.

Ottosen, N.S., Ristinmaa, M., Ljung, C., 2000. Rayleigh waves obtained by the indeterminate couple-stress theory. European Journal of Mechanics-A/Solids 19, 929-947.

Park, S., Gao, X., 2006. Bernoulli-euler beam model based on a modified couple stress theory. Journal of Micromechanics and Microengineering 16, 2355.

Radi, E., Bianchi, G., Nobili, A., 2020. Bounds to the pull-in voltage of a mems/nems beam with surface elasticity. Applied Mathematical Modelling $91,1211-1226$. 
Sengupta, P., Ghosh, B., 1974. Effect of couple-stresses on the propagation of waves in an elastic layer. Pure and applied geophysics 112, 331-338.

Sharma, V., Kumar, S., 2014. Velocity dispersion in an elastic plate with microstructure: effects of characteristic length in a couple stress model. Meccanica 49, 1083-1090.

Toupin, R., 1962. Elastic materials with couple-stresses. Archive for rational mechanics and analysis 11, 385-414.

Tsiatas, G., 2009. A new Kirchhoff plate model based on a modified couple stress theory. International Journal of Solids and Structures 46, 2757-2764.

Wang, C., Chen, X., Wei, P., Li, Y., 2017. Reflection and transmission of elastic waves through a couple-stress elastic slab sandwiched between two half-spaces. Acta Mechanica Sinica 33, 1022-1039.

Yang, F., Chong, A., Lam, D., Tong, P., 2002. Couple stress based strain gradient theory for elasticity. International journal of solids and structures 39, 2731-2743.

Yin, L., Qian, Q., Wang, L., Xia, W., 2010. Vibration analysis of microscale plates based on modified couple stress theory. Acta Mechanica Solida Sinica 23, 386-393.

Zhou, S.S., Gao, X.L., 2014. A nonclassical model for circular Mindlin plates based on a modified couple stress theory. Journal of Applied Mechanics 81. 